\title{
Approximation of the Viability Kernel
}

\author{
Patrick Saint-Pierre \\ CEREMADE, Université Paris-Dauphine \\ Place du Maréchal de Lattre de Tassigny \\ 75775 Paris cedex 16
}

26 october 1990

\begin{abstract}
We study recursive inclusions $x^{n+1} \in G\left(x^{n}\right)$. For instance such systems appear for discrete finite difference inclusions $x^{n+1} \in G_{\rho}\left(x^{n}\right)$ where $G_{\rho}:=\mathbf{1}+\rho F$. The discrete viability kernel of $G_{\rho}$, i.e. the largest discrete viability domain, can be an internal approximation of the viability kernel of $K$ under $F$. We study discrete and finite dynamical systems. In the Lipschitz case we get a generalization to differential inclusions of Euler and Runge-Kutta methods. We prove first that the viability kernel of $K$ under $F$ can be approached by a sequence of discrete viability kernels :associated with $\Gamma_{\rho}(x)=x+\rho F(x)+\frac{M l}{2} \rho^{2} \mathcal{B}$. Secondly, we show that it can be approached by finite viability kernels associated with $\Gamma_{h \rho}^{\alpha}(x):=x+\rho F(x): x_{h}^{n+1} \in\left(\Gamma_{h \rho}\left(x_{h}^{n}\right)+\alpha(h) \mathcal{B}\right) \cap X_{h}$.
\end{abstract}

\section{Introduction}

Let $X$ a finite dimentional vector space and $K$ a compact subset of $X$.

Let us consider the differential inclusion:

$$
\left\{\begin{array}{l}
\dot{x}(t) \in F(x(t)), \text { for almost all } t \geq 0, \\
x(0)=x_{0} \in K,
\end{array}\right.
$$

where $F$ is a Marchaud man ${ }^{1}$ defined from $K$ to $X$.

With this inclusion, for a fixed $\rho>0$, we associate the discrete explicit scheme:

$$
\left\{\begin{array}{l}
\frac{x^{n+1}-x^{n}}{\rho} \in F\left(x^{n}\right), \text { for all } n \geq 1, \\
x^{0}=x_{0} \in K,
\end{array}\right.
$$

We note $G_{\rho}$ the set-valued map $G_{\rho}=\mathbf{1}+\rho F$ and the system (2) can be rewrited as follows:

$$
x^{n+1} \in G_{\rho}\left(x^{n}\right), \text { for all } n \geq 0,
$$

\footnotetext{
${ }^{1}$ A set-valued map $F: X \rightsquigarrow Y$ is a Marchaud map if

$$
\left\{\begin{array}{l}
\operatorname{Dom}(F) \neq \emptyset \\
F \text { is upper-semicontinuous, convex compact valued }
\end{array}\right.
$$$$
\forall x \in \operatorname{Dom}(F),\|F(x)\|:=\max _{y \in F(x)}\|y\| \leq c(\|x\|+1)
$$ 
The Viability Theory allows to study viable solutions of (1) and the subset of elements $x_{0} \in K$ such that there exists at least a viable solution starting at $x_{0}$. On the other hand, we look for approximation of such solutions and we wonder how the set of initial points from which there exists at least a viable approximation solution to (2) and the set of initial points from which there exists at least a viable solution to (1) are related together.

These sets are called viability kernel of $K$ under $F$ or discrete viability kernel of $K$ under $G \rho$. Byrnes \& Isidori [5] and Frankowska \& Quincampoix [8] have proposed algorithms which approximate the viability kernel of $K$ under $F$ when $F$ is lipschitzian and $K$ is closed.

We prove that, when $F$ is a Marchaud map, for a good choice of discretizations $G \rho$, the sequence of discrete viability kernels of $K$ under $G \rho$ converges to a subset contained in the viability kernel of $K$ under $F$. Moreover it converges to the viability kernel if $F$ is lipschitzian.

We show that similar results remain true when we introduce a discretization of the space and consider finite viability kernels.

\section{Definitions and General Results}

We call discrete dynamical system associated with $G$ the following system:

$$
x^{n+1} \in G\left(x^{n}\right), \text { for all } n \geq 0,
$$

We denote by

- $\mathcal{K}$ the set of all sequences from $\mathbb{N}$ to $K$.

- $\vec{x}:=\left(x^{0}, \ldots, x^{n}, \ldots\right) \in \mathcal{X}$ a solution to discrete dynamical system (4)

- $\vec{S}_{G}\left(x^{0}\right)$ the set of solutions $\vec{x} \in \mathcal{X}$ to the discrete dynamical system starting at $x_{0}$.

A solution $\vec{x}$ is viable if and only if $\vec{x} \in \vec{S}_{G}(x) \cap \mathcal{K}$ :

$$
\left\{\begin{array}{l}
x^{n+1} \in G\left(x^{n}\right), \quad \forall n \geq 0, \\
x^{0}=x \in K \\
x^{n} \in K, \quad \forall n \geq 0 .
\end{array}\right.
$$

It means that there exists a selection of equation (2) which remains in $K$ at each step $n$.

We study the subset of initial points in $K$ from which there exists at least one viable solution.

Definition 2.1 Let $G: X \rightsquigarrow X$ be a set-valued map. A subset $D \subset X$ is a discrete viability domain of $G$ if

$$
\forall x \in D, G(x) \cap D \neq \emptyset
$$

Let $K$ be a subset of $X$. The discrete viability kernel of $K$ under $G$ is the largest closed discrete viability domain contained in $K$ and we denote it $\operatorname{Viab}_{G}(K)$. 
We can point out the following remark and properties:

$$
\operatorname{Viab}_{G}(K)=\left\{x \in K, \text { such that } \vec{S}_{G}(x) \cap \mathcal{K} \neq \emptyset\right\}
$$

Since $\operatorname{Viab}_{G}(K)$ is the largest discrete viability domain contained in $K$, any solution of (5) starting from any initial point $x_{0} \in K \backslash V i a b_{G}(K)$ never meets the discrete viability kernel $\operatorname{Viab}_{G}(K)$ while it remains in $K$.

Moreover any solution of (5) which does not start from $\operatorname{Viab}_{G}(K)$ must leave $\mathrm{K}$ in a finite number of steps.

For all closed $K_{1}, K_{2}$ such that $K_{1} \subset K_{2} \subset X$, then

$$
\operatorname{Viab}_{G}\left(K_{1}\right) \subset \operatorname{Viab}_{G}\left(K_{2}\right) \subset X
$$

For all setvalued maps $G_{1}, G_{2}$ such that $\forall x \in K: G_{1}(x) \subset G_{2}(x)$, then

$$
\operatorname{Viab}_{G_{1}}(K) \subset \operatorname{Viab}_{G_{2}}(K) \subset X
$$

For all subset $K^{\prime}$ such that $\operatorname{Viab}_{G}(K) \subset K^{\prime} \subset K$, then

$$
\operatorname{Viab}_{G}\left(K^{\prime}\right)=\operatorname{Viab}_{G}(K)
$$

\subsection{A Construction Method for Discrete Viability Kernel}

Let us consider the sequence of subsets $K^{0}=K, K^{1}, \ldots, K^{n}, \ldots$ defined as follows:

$$
K^{n+1}:=\left\{x \in K^{n} \text { such that: } G(x) \cap K^{n} \neq \emptyset\right\}
$$

We note

$$
K^{\infty}:=\bigcap_{n=0}^{+\infty} K^{n}
$$

Proposition 2.1 Let $G$ : $X \rightsquigarrow X$ a upper semicontinuous set-valued map with closed values and $K$ a compact subset of $\operatorname{Dom}(G)$. Then

$$
K^{\infty}=\operatorname{Viab}_{G}(K)
$$

Proof - Let us prove that $\forall n \in \mathbb{N}, \operatorname{Viab}_{G}(K) \subset K^{n}$. We have $\operatorname{Viab}_{G}(K) \subset K^{0}$. Since $G$ is upper semicontinuous,

$$
K^{1}=\left\{x \in K^{0}, G(x) \cap K^{0} \neq \emptyset\right\}
$$

is closed and, for all $x \in K^{0} \backslash K^{1}$, it does not exit any viable solution starting from $x$. This implies recursively that

$$
\operatorname{Viab}_{G}(K) \cap\left(K^{0} \backslash K^{1}\right)=\emptyset
$$

and then:

$$
\operatorname{Viab}_{G}(K) \subset K^{1} \subset K
$$


Let us assume that

$$
\operatorname{Viab}_{G}(K)=\operatorname{Viab}_{G}\left(K^{n-1}\right) \subset K^{n-1} .
$$

Since

$$
K^{n}=\left\{x \in K^{n-1}, G(x) \cap K^{n-1} \neq \emptyset\right\},
$$

for all $x \in K^{n-1} \backslash K^{n}$, it does not exist any solution starting from $x$ viable in $K^{n-1}$, and thus in $K$. Then $\operatorname{Viab}_{G}(K) \cap\left(K^{n-1} \backslash K^{n}\right)=\emptyset$ and $\operatorname{Viab}_{G}(K)=$ $\operatorname{Viab}_{G}\left(K^{n}\right) \subset K^{n}$. This implies that

$$
\operatorname{Viab}_{G}(K) \subset K^{\infty} .
$$

Conversely, from definition 2.1, $K^{\infty}$ is a viability domain: indeed for any $x \in$ $K^{\infty}, \forall n \in \mathbb{N}: x \in K^{n+1}$ and then $G(x) \cap K^{n} \neq \emptyset$. Since for any fixed $x$, sets $G(x) \cap K^{n}$ form a decreasing sequence of non empty compact subsets, then $G(x) \cap K^{\infty}$ is non empty. We have proved that $K^{\infty}$ is a viability domain of $G$ and since $\operatorname{Viab}_{G}(K)$ is the largest viability domain:

$$
K^{\infty} \subset \operatorname{Viab}_{G}(K) .
$$

Definition 2.2 Let $G: X \rightsquigarrow X$ a set-valued map and $r>0$. We call extension of $G$ with a ball of radius $r$ the set-valued map $G^{r}: X \rightsquigarrow X$ defined by:

$$
G^{r}(x):=G(x)+r \mathcal{B}
$$

We consider the sequence of subsets $K^{r, 0}=K, K^{r, 1}, \ldots, K^{r, n}, \ldots$ defined as follows:

(12) $K^{r, n+1}:=\left\{x \in K^{r, n}\right.$ such that $\left.G^{r}(x) \cap K^{r, n} \neq \emptyset\right\}, K^{r, \infty}:=\bigcap_{n=0}^{+\infty} K^{r, n}$

If $G$ is an upper semicontinuous set-valued map, $G^{r}: X \rightsquigarrow X$ is also upper semicontinuous and from Proposition 2.1

$$
\forall r>0, \quad K^{r, \infty}=\operatorname{Viab}_{G^{r}}(K)
$$

When $r$ decreases to 0 , the viability kernel of $K$ under $G^{r}$ converges to the viability kernel of $K$ under $G$ :

Proposition 2.2 Let $G$ be upper semicontinuous and $K$ a compact subset of $X$. The following property holds:

$$
\operatorname{Viab}_{G}(K)=\bigcap_{r>0} \operatorname{Viab}_{G^{r}}(K)
$$


Proof - Let $x_{0} \in \bigcap_{r>0} \operatorname{Viab}_{G^{r}}(K)$. For all $r>0$, Proposition 2.1 implies:

$$
G^{r}\left(x_{0}\right) \cap \operatorname{Viab}_{G^{r}}(K) \neq \emptyset, \quad \forall r>0
$$

$G^{r}\left(x_{0}\right)$ is closed, $\operatorname{Viab}_{G^{r}}(K)$ is compact and both are, from (8), decreasing sets when $r$ decreases to zero. Also the intersection $G^{r}\left(x_{0}\right) \cap V i a b_{G^{r}}(K)$ is a decreasing sequence of nonempty compact sets and

$$
\bigcap_{r>0}\left(G^{r}\left(x_{0}\right) \cap \operatorname{Viab}_{G^{r}}(K)\right)=G\left(x_{0}\right) \cap\left(\bigcap_{r>0} \operatorname{Viab}_{G^{r}}(K)\right) \neq \emptyset
$$

Then $\bigcap_{r>0} \operatorname{Viab}_{G^{r}}(K)$ is a viability domain of $G$. Since $\operatorname{Viab}_{G}(K)$ is the viability kernel of $G$, from definition 2.1 it contains $\bigcap_{r>0} \operatorname{Viab}_{G^{r}}(K)$.

When $G$ is a $k$-Lipschitz setvalued map, we have the following result giving an estimation of the growth of the discrete viability kernel when $r$ increases:

Proposition 2.3 Let $G: X \rightsquigarrow X$ a $k$-Lipschitz set-valued map, $K$ a closed subset of $X$. Let $G^{r}:=G+r \mathcal{B}, \operatorname{Viab}_{G^{r}}(K)$ and $\operatorname{Viab}_{G}(K)$ the discrete viability kernel of $G^{r}$ and $G$ respectively.

Then:

(see footnot (2) $^{2}$

$$
\operatorname{Viab}_{G}(K)+\frac{r}{k} \mathcal{B} \subset \operatorname{Viab}_{G^{r}}(K), \quad \forall r>0
$$

Proof - Let $r>0$ given, $x \in \operatorname{Viab}_{G}(K)$ arbitrairely choosen, $\eta<$ $\min \left(\eta_{0}, \frac{r}{k}\right)$ and $x^{\prime} \in(\{x\}+\eta \mathcal{B}) \cap K$. Then

$$
\left\{\begin{array}{lll}
\text { i) } & \forall x \in \operatorname{Viab}_{G}(K) & G(x) \cap \operatorname{Viab}_{G}(K) \neq \emptyset \\
\text { ii) } & \forall x \in \operatorname{Viab}_{G^{r}}(K) & G^{r}(x) \cap \operatorname{Viab}_{G^{r}}(K) \neq \emptyset
\end{array}\right.
$$

Since $G$ is $k$-Lipschitz and $k \eta<r$,

$$
G(x) \subset G\left(x^{\prime}\right)+k\left\|x-x^{\prime}\right\| \mathcal{B} \subset G^{r}\left(x^{\prime}\right)
$$

From 16, we deduce that

$$
G^{r}\left(x^{\prime}\right) \cap \operatorname{Viab}_{G}(K) \neq \emptyset
$$

and since $\operatorname{Viab}_{G}(K) \subset \operatorname{Viab}_{G^{r}}(K)$

$$
G^{r}\left(x^{\prime}\right) \cap \operatorname{Viab}_{G^{r}}(K) \neq \emptyset
$$

Then $\forall x \in \operatorname{Viab}_{G}(K), \forall x^{\prime} \in(\{x\}+\eta \mathcal{B}) \cap K$, there exists a viable solution for the system associated with $G^{r}$ starting from $x^{\prime}$ and thus $x^{\prime} \in V i a b_{G^{r}}(K)$.

\footnotetext{
2 with the convention: $\emptyset+\eta \mathcal{B}=\emptyset$
} 


\section{Approximation of Viability Kernels for Finite Difference Inclusions}

Let $F$ a Marchaud map and $\Gamma_{\rho}$ a sequence of setvalued maps which correspond to discretizations associated with the initial differential inclusion (1) satisfying:

$$
\left.\left.\forall \epsilon>0, \exists \rho_{\epsilon}>0, \forall \rho \in\right] 0, \rho_{\epsilon}\right]: \operatorname{Graph}\left(\frac{\Gamma_{\rho}-\mathbf{1}}{\rho}\right) \subset \operatorname{Graph}(F)+\epsilon \mathcal{B}
$$

where $\mathcal{B}$ is the unit ball in $X \times X$.

We note

$$
F_{\rho}:=\frac{\Gamma_{\rho}-\mathbf{1}}{\rho} .
$$

Assumption (17) implies that the graph of $F$ contains the graphical upper limit ${ }^{3}$ of $F_{\rho}$, that is to say that $\operatorname{Graph}(F)$ contains the Painlevé-Kuratowski upper limit 4 of $\operatorname{Graph}\left(F_{\rho}\right)$ :

$$
\limsup _{\rho \rightarrow 0} \operatorname{Graph}\left(F_{\rho}\right) \subset \operatorname{Graph}(F)
$$

Let $K_{\rho}$ a sequence of subsets of $X$ such that $K=\lim \sup _{\rho>0} K_{\rho}$. Possible $K_{\rho}$ may be constant.

Let $\operatorname{Viab}_{\Gamma_{\rho}}\left(K_{\rho}\right)$ the discrete viability kernel of $K_{\rho}$ under $\Gamma_{\rho}$.

\subsection{The Viability Kernel Convergence Theorem}

Theorem 3.1 Let $F$ a Marchaud map and $\Gamma_{\rho}$ a sequence of set-valued maps such that $F=\overline{C o} \operatorname{Lim}_{\rho \rightarrow 0}^{\sharp}\left(\frac{\Gamma_{\rho}-\mathbf{1}}{\rho}\right)$. Then the upper limit $K^{\sharp}=\lim \sup _{\rho \rightarrow 0} \operatorname{Viab}_{\Gamma_{\rho}}\left(K_{\rho}\right)$ is a viable subset under $F$ :

$$
\limsup _{\rho \rightarrow 0} \operatorname{Viab}_{\Gamma_{\rho}}\left(K_{\rho}\right) \subset \operatorname{Viab}_{F}(K)
$$

Proof - Let us consider $x_{0} \in K^{\sharp}$. There exists a subsequence $x_{\rho, 0} \in \operatorname{Viab}_{\Gamma_{\rho}}\left(K_{\rho}\right)$ which converges to $x_{0}$ and a $K_{\rho}$-viable solution $\vec{x}_{\rho}:=\left(x_{\rho}^{0}, \ldots, x_{\rho}^{n}, \ldots\right) \in \vec{S}_{\Gamma_{\rho}}\left(x_{\rho}^{0}\right) \cap$ $\mathcal{K}_{\rho}$ to the discrete system associated with $\Gamma_{\rho}$.

From the definition of $\Gamma_{\rho}, x_{\rho}^{n+1} \in \Gamma_{\rho}\left(x_{\rho}^{n}\right)$ and then:

$$
\forall n>0, \frac{x_{\rho}^{n+1}-x_{\rho}^{n}}{\rho} \in F_{\rho}\left(x_{\rho}^{n}\right)
$$

\footnotetext{
${ }^{3}$ The graphical upper limit is the upper limit of the sequence of $\operatorname{Graph}\left(F_{\rho}\right)$.

${ }^{4}$ The upper limit of a sequence of subsets $D_{n}$ of $X$ is

$$
D^{\sharp}=\limsup _{n \rightarrow \infty} D_{n}:=\left\{y \in X \mid \liminf _{n \rightarrow \infty} d\left(y, D_{n}\right)=0\right\}
$$
}


With this sequence we associate the piecewise linear interpolation $x_{\rho}(\cdot)$ which coincides to $x_{\rho}^{n}$ at nodes $n \rho$ :

$$
x_{\rho}(t)=x_{\rho}^{n}+\frac{x_{\rho}^{n+1}-x_{\rho}^{n}}{\rho}(t-n \rho), \forall t \in[n \rho,(n+1) \rho[, \forall n>0
$$

Then

$$
\dot{x}_{\rho}(t) \in F_{\rho}\left(x_{\rho}^{n}\right), \forall t \in[n \rho,(n+1) \rho[
$$

We have

$$
d\left(\left(x_{\rho}(t), \dot{x}_{\rho}(t)\right), \operatorname{Graph}\left(F_{\rho}\right)\right) \leq\left\|x_{\rho}(t)-x_{\rho}^{n}\right\| \leq \rho\left\|F\left(x_{\rho}^{n}\right)\right\|
$$

Since $F$ is Marchaud, and from (18), set-valued maps $F_{\rho}$ satisfy a uniform linear growth:

$$
\exists c>0,\left\|F_{\rho}(x)\right\| \leq c(\|x\|+1), \forall x \in X
$$

As in the proof of the Viability Theorem (see [2, [9]), this implies

$$
\left\{\begin{array}{lll}
\forall t>0, & \left.\left\|x_{\rho}(t)\right\| \leq\left(\| x_{0}\right) \|+1\right) e^{c t} \\
\text { for almost all } t>0, & \left\|x_{\rho}^{\prime}(t)\right\| \leq c\left(\left\|x_{0}\right\|+1\right) e^{c t}
\end{array}\right.
$$

Then, $\forall \epsilon>0, \forall t>0$, there exists $\rho_{\epsilon, t}>0$ such that

$$
\left.\forall \rho \in] 0, \rho_{\epsilon, t}\right], d\left(\left(x_{\rho}(t), \dot{x}_{\rho}(t)\right), \operatorname{Graph}\left(F_{\rho}\right)\right) \leq c\left(\left\|x_{0}\right\|+1\right) e^{c t} \leq \frac{\epsilon}{2}
$$

and with 17) we have

$$
\left.\left.\exists \rho_{\epsilon}^{\prime}>0, \forall \rho \in\right] 0, \rho_{\epsilon}^{\prime}\right]: \operatorname{Graph}\left(F_{\rho}\right) \subset \operatorname{Graph}(F)+\frac{\epsilon}{2} \mathcal{B}
$$

Let $\rho_{\epsilon, t}^{0}:=\min \left(\rho_{\epsilon}^{\prime}, \rho_{\epsilon, t}\right)>0$ then

$$
\left.\left.\left(x_{\rho}(t), \dot{x}_{\rho}(t)\right) \in \operatorname{Graph}(F)+\epsilon \mathcal{B}, \quad \forall \rho \in\right] 0, \rho_{\epsilon, t}\right]
$$

By the Ascoli and Alaoglu Theorems, we derive that there exists $x(\cdot) \in W^{1,1}\left(0,+\infty ; X ; e^{-c t} d t\right)$ and a subsequence (again denoted by) $x_{\rho}$ which satisfy:

$$
\begin{cases}\text { i) } & x_{\rho}(\cdot) \text { converges uniformly to } x(\cdot) \\ \text { ii) } & x_{\rho}^{\prime}(\cdot) \text { converges weakly to } x^{\prime}(\cdot) \text { in } L^{1}\left(0,+\infty ; X ; e^{-c t} d t\right)\end{cases}
$$

This implies (see [1] The Convergence Theorem) that $x(\cdot)$ is a solution to the differential inclusion:

$$
\left\{\begin{array}{l}
\dot{x}(t) \in F(x(t)), \text { for almost all } t \geq 0 \\
x(0)=x_{0} \in K
\end{array}\right.
$$

It remains to prove that the limit is a viable solution:

$\forall t>0$, there exists a sequence $n_{t}=E\left(\frac{t}{\rho}\right)$ such that $n_{t} \rho \rightarrow t$ when $\rho \rightarrow 0$. Then $x(t)=\lim _{\rho \rightarrow 0} x_{\rho}\left(n_{t} \rho\right)$. Since $\forall \rho: x_{\rho}\left(n_{t} \rho\right)=x_{\rho}^{n_{t}} \in \operatorname{Viab}_{\Gamma_{\rho}}\left(K_{\rho}\right), x(t)$ belongs to the upper limit $K^{\sharp}$ of subsets $\operatorname{Viab}_{\Gamma_{\rho}}\left(K_{\rho}\right) \subset K_{\rho}$ and then $K^{\sharp} \subset K$. This implies that $K^{\sharp} \subset \operatorname{Viab}_{F}(K)$. 


\subsection{Examples of Approximation Processes}

\section{1 - The finite difference explicit scheme.}

Naturally, the discrete explicit scheme (2)

$$
\left\{\begin{array}{l}
\frac{x^{n+1}-x^{n}}{\rho} \in F\left(x^{n}\right), \text { for all } n \geq 1, \\
x^{0}=x_{0} \in K,
\end{array}\right.
$$

is associated with $F_{\rho}=F, K_{\rho}=K$ and $G_{\rho}:=\mathbf{1}+\rho F$. It already satisfies property 17) for any $\epsilon$.

\section{2 - The set-valued Runge-Kutta method.}

Let us define the set-valued Runge-Kutta scheme $\Gamma_{\rho}^{R K}$ :

For any $x \in K$,

$$
F_{\rho}^{R K}(x):=\left\{y \in X \mid y=\frac{1}{2}(\beta+\gamma)-\frac{\rho}{2} \beta \text { where } \beta \in F(x), \gamma \in F(x+\rho \beta)\right\}
$$

and

$$
\Gamma_{\rho}^{R K}(x):=\mathbf{1}+\rho F_{\rho}^{R K}(x)
$$

Let $(x, y) \in \operatorname{Graph}\left(\Gamma_{\rho}^{R K}\right)$. From definition $(20)$, there exist $\beta \in F(x)$ and $\gamma \in F(x+\rho \beta)\}$ such that $y=\frac{1}{2}(\beta+\gamma)-\frac{\rho}{2} \beta$. Since $F(x)$ is Marchaud, $\|\beta\|$ is bounded by $m$ and since $F$ is upper semicontinuous,

$$
\left.\left.\forall \epsilon>0, \exists \rho_{\epsilon, m}, \forall \rho \in\right] 0, \rho_{\epsilon, m}\right], F(x+\rho \beta) \subset F(x)+\epsilon \mathcal{B} .
$$

Since $F$ is convex valued, $\frac{1}{2}(\beta+\gamma) \in F(x)+\frac{\epsilon}{2} \mathcal{B}$.

Then, choosing $\rho \leq \min \left(\rho_{\epsilon}, \frac{\epsilon}{2 m}\right)$, we have $F_{\rho}^{R K}(x) \subset F(x)+\epsilon \mathcal{B}$ and then

$$
\operatorname{Graph}\left(\frac{\Gamma_{\rho}^{R K}(x)-\mathbf{1}}{\rho}\right) \subset \operatorname{Graph}(F(x))+\epsilon \mathcal{B}
$$

Then condition (17) holds and from Theorem 3.1 we deduce the following corollary:

Corollary 3.1 The upper limit of $\operatorname{Viab}_{\Gamma_{\rho}^{R K}}(K)$ is a viable subset under F:

$$
\limsup _{\rho \rightarrow 0} \operatorname{Viab}_{\Gamma_{\rho}^{R K}}(K) \subset \operatorname{Viab}_{F}(K)
$$

\section{3 - The thickening process.}

Let us define the set-valued map $F_{\rho}^{T}: X \rightarrow X$ by a thickening of the values of $F$ by balls of radius $\frac{M l}{2} \rho$ :

$$
F_{\rho}^{T}(x)=F(x)+\frac{M l}{2} \rho \mathcal{B}
$$


and consider the set-valued map associated with the finite differnece scheme for $F_{\rho}^{T}$ :

$$
\Gamma_{\rho}^{T}(x)=x+\rho F_{\rho}^{T}(x)
$$

When $F$ is Marchaud, we have the following relations between $\operatorname{Viab}_{G_{\rho}}(K)$, $\operatorname{Viab}_{\Gamma_{\rho}^{T}}(K)$ and $\operatorname{Viab}_{F}(K)$ :

Corollary 3.2 Let $F$ a Marchaud map, $G_{\rho}$ and $\Gamma_{\rho}$ defined by (22).

Then

$$
\limsup _{\rho \rightarrow 0} V i a b_{G_{\rho}}(K) \subset \underset{\rho \rightarrow 0}{\limsup } \operatorname{Viab}_{\Gamma_{\rho}^{T}}(K) \subset V i a b_{F}(K)
$$

Proof - The first inclusion holds true since $G_{\rho}(x) \subset \Gamma_{\rho}^{T}(x)$ and 8 . On the other hand, since

$$
\operatorname{Graph}\left(\frac{\Gamma_{\rho}^{T}-\mathbf{1}}{\rho}\right)=\operatorname{Graph}\left(F_{\rho}^{T}\right) \subset \operatorname{Graph}(F)+\frac{M l}{2} \rho \mathcal{B}
$$

Theorem 3.1 implies the second inclusion.

\subsection{Approximation of the Viability Kernel in the Lipschitz case}

From now on we use the following notations :

$$
\begin{aligned}
& G_{\rho}=\mathbf{1}+\rho F \\
& F_{\rho}=F+\frac{M l}{2} \rho \mathcal{B} \\
& \Gamma_{\rho}=\mathbf{1}+\rho F_{\rho}=\mathbf{1}+\rho F+\frac{M l}{2} \rho^{2} \mathcal{B}
\end{aligned}
$$

When $F$ is $l$-Lipschitz, we claim that the discrete viability kernel $\operatorname{Viab}_{\Gamma_{\rho}}(K)$ is a good approximation of the viability kernel of $K$ under $F$.

Theorem 3.2 5 Let $F$ a Marchaud and l-Lipschitz set-valued map, let $K$ a closed subset of $X$ satisfying the boundedness condition

$$
M:=\sup _{x \in K} \sup _{y \in F(x)}\|y\|<\infty
$$

Then

$$
\limsup _{\rho \rightarrow 0} \operatorname{Viab}_{\Gamma_{\rho}}(K)=\operatorname{Viab}_{F}(K)
$$

\footnotetext{
${ }^{5}$ This result is due to M. Quincampoix and the author when they visit IIASA Institute Laxenburg, Austria
} 
Proof - Since $F$ is Marchaud, from Corollary 3.2 .

$$
\limsup _{\rho \rightarrow 0} \operatorname{Viab}_{\Gamma_{\rho}}(K) \subset \operatorname{Viab}_{F}(K)
$$

We want to check the opposite inclusion.

Let $x_{0} \in K$ and consider any solution $x(\cdot) \in \mathcal{S}_{F}\left(x_{0}\right)$. Let $\rho>0$ given. We have

$$
x(t+\rho)-x(t)=\int_{t}^{t+\rho} \dot{x}(s) d s, \forall t>0
$$

$\dot{x}(s) \in F(x(s))$ and $F$ Lipschitzian imply that

$$
x(t+\rho)-x(t) \in \rho F(x(t))+l \int_{t}^{t+\rho}\|x(s)-x(t)\| d s \mathcal{B}, \forall t>0
$$

But since $F$ is bounded, $\|x(s)-x(t)\| \leq(s-t) M$ and thus

$$
x(t+\rho)-x(t) \in \rho F(x(t))+\frac{M l}{2} \rho^{2} \mathcal{B}
$$

So, we have proved that if $x(\cdot) \in S_{F}\left(x_{0}\right)$ then the following sequence

$$
\xi_{n}=x(n \rho), \quad \forall n \geq 0
$$

is a solution to the discrete dynamical system associated with $\Gamma_{\rho}$ :

$$
\xi_{n+1} \in \Gamma_{\rho}\left(\xi_{n}\right), \quad \forall n \geq 0
$$

Moreover, if $x(\cdot)$ is a viable solution, then $\left(\xi_{n}\right)_{n}$ is a viable solution to (30). Thus

$$
\operatorname{Viab}_{F}(K) \subset \operatorname{Viab}_{\Gamma_{\rho}}(K), \quad \forall \rho>0
$$

and then

$$
\operatorname{Viab}_{F}(K) \subset \limsup _{\rho \rightarrow 0^{+}} \operatorname{Viab}_{\Gamma_{\rho}}(K) .
$$

\section{Approximation by Finite Setvalued Maps}

With any $h \in \mathbb{R}$ we associate $X_{h}$ a countable subset of $X$, which spans $X$ in the sense that

$$
\forall x \in X, \exists x_{h} \in X_{h} \text { such that }\left\|x-x_{h}\right\| \leq \alpha(h)
$$

where $\alpha(h)$ decreases to 0 when $h \rightarrow 0$ :

$$
\lim _{h \rightarrow 0} \alpha(h)=0
$$




\subsection{Approximation of discrete and finite viability kernels}

Let $G_{h}: X_{h} \rightsquigarrow X_{h}$ a finite set-valued map and a subset $K_{h} \subset \operatorname{Dom}\left(G_{h}\right)$.

We call finite dynamical system associated with $G_{h}$ the following system:

$$
x_{h}^{n+1} \in G_{h}\left(x_{h}^{n}\right), \text { for all } n \geq 0,
$$

and we denote by

- $\mathcal{K}_{h}$ the set of all sequences from $\mathbb{N}$ to $K_{h}$.

- $\vec{x}_{h}:=\left(x_{h}^{0}, \ldots, x_{h}^{n}, \ldots\right) \in \mathcal{X}_{h}$ a solution to system 35

- $\vec{S}_{G_{h}}\left(x_{h}^{0}\right)$ the set of solutions $\vec{x}_{h} \in \mathcal{X}_{h}$ to the finite differential inclusion 35 starting from $x_{h}^{0}$

A solution $\vec{x}_{h}$ is viable if and only if $\vec{x}_{h} \in \vec{S}_{G_{h}}\left(x_{h}\right) \cap \mathcal{K}_{h}$, that is to say that:

$$
\left\{\begin{array}{l}
x_{h}^{n+1} \in G_{h}\left(x_{h}^{n}\right), \quad \forall n \geq 0, \\
x_{h}^{0}=x_{h} \in K_{h} \\
x_{h}^{n} \in K_{h}, \quad \forall n \geq 0 .
\end{array}\right.
$$

Let $K_{h}^{0}=K_{h}, K_{h}^{1}, \ldots, K_{h}^{n}, \ldots$ defined recursively as in the second section:

$$
K_{h}^{n+1}:=\left\{x_{h} \in K_{h}^{n} \text { such that: } G_{h}\left(x_{h}\right) \cap K_{h}^{n} \neq \emptyset\right\}
$$

The viability kernel algorithm and Proposition 2.1 holds true for finite dynamical systems whenever the set-valued map $G_{h}$ has nonempty values and we have:

$$
\operatorname{Viab}_{G_{h}}\left(K_{h}\right)=K_{h}^{\infty}:=\bigcap_{n=0}^{+\infty} K_{h}^{n}
$$

Let us notice that $K_{h}^{\infty}$ can be emptyset and in any case there exists a finite integer $p$ such that:

$$
K_{h}^{\infty}=K_{h}^{n}=K_{h}^{p}, \forall n>p
$$

What happen when $G_{h}$ is the reduction to $K_{h}$ of a set-valued map $G$ ?

We cannot apply no longer more Proposition 2.1 since $G\left(x_{h}\right)$ may not contain any point of the reduction $X_{h}$ of $X$ and $G_{h}\left(x_{h}\right)$ be empty.

To turnover this difficulty, we will consider greater set-valued maps $G^{r}$ which still approximate $G$. But the choice of such approximations is subjet to two opposite considerations: on one hand, they have to be large enough in order that the reductions to $X_{h}$ of such approximations have their domain containing $K_{h}$ (have nonempty values on $K_{h}$ ), and so, it will be possible to apply again Proposition 2.1. On the other hand, the enlargement is limited as far as the graphical assumption (1) of Theorem 3.1 still holds so as to the viability kernel of $K$ under $G$ contains the upper limit of finite viability kernels of $K_{h}$ under the finite set-valued approximations $G_{h}^{r}$.

In case of upper semicontinuous set-valued maps, we bring in the fore some discretization process which leads to approach a subset of a the viability kernel.

In the Lipschitz case, these process enables us to approach the viability kernel completely. 


\section{Notations:}

the reduction to the finite subset $X_{h}$ of any subset $D$ will be noted by a lower index $h: D_{h}:=D \cap X_{h}$;

the extension of a set-valued map $G$ with a ball of radius $r$ by an upper index: $\forall x \in X, G^{r}(x):=G(x)+r \mathcal{B}$.

Thus the reduction to $X_{h}$ of the extension of a set-valued map $G$ with a ball of radius $r$ will be noted $G_{h}^{r}$.

Let us notice that the extension operation has to be done before the reduction one otherwise it could be empty even for $r>\alpha(h)$.

From property 33 which defines $\alpha(h)$, we consider now the extension with $r=\alpha(h)$. We observe that $G_{h}^{\alpha(h)}$ satisfies the non emptyness property:

$$
\forall x_{h} \in \operatorname{Dom}(G) \cap X_{h}, G_{h}^{\alpha(h)}\left(x_{h}\right):=G^{\alpha(h)}\left(x_{h}\right) \cap X_{h} \neq \emptyset
$$

and the decreasing sequence of finite subsets $K_{h}^{\alpha(h), 0}=K_{h}, K_{h}^{\alpha(h), 1}, \ldots, K_{h}^{\alpha(h), n}, \ldots$ defined by

$$
K_{h}^{\alpha(h), n+1}:=\left\{x \in K_{h}^{\alpha(h), n} \text { such that } G_{h}^{\alpha(h)}(x) \cap K_{h}^{\alpha(h), n} \neq \emptyset\right\}
$$

satisfies property 37 :

$$
K_{h}^{\alpha(h), \infty}:=\bigcap_{n=0}^{+\infty} K_{h}^{\alpha(h), n}=\operatorname{Viab}_{G_{h}^{\alpha(h)}}\left(K_{h}\right)
$$

As a partial conclusion, we are able to approximate the discrete viability kernel of $K$ under $G$ : first we extend $G$ such that for all $x \in K$, images of $G^{r}$ encounters $X_{h}$, in other words such that $\operatorname{Dom}\left(G_{h}^{r}\right)=\operatorname{Dom}(G) \cap X_{h}$. To be sure of this, without loss of generality, we can choose $r=\alpha(h)$. Secondly we look after the discrete viability kernel of $K$ under $G^{r}$, the finite viability kernel of $K_{h}$ under $G_{h}^{r}$ and at last we let $h$ decreasing to 0 .

What relations link together the discrete viability kernels $\operatorname{Viab}_{G}(K)$ or $\operatorname{Viab}_{G^{\alpha(h)}}(K)$ and the finite viability kernels $\operatorname{Viab}_{G_{h}}\left(K_{h}\right)$ or $\operatorname{Viab}_{G_{h}^{\alpha(h)}}\left(K_{h}\right)$ whenever $K_{h}$ is the reduction of $K$ to $X_{h}$ : are the latters the reduction to $X_{h}$ of the formers? Does the upper limit of the latters, when $h$ goes to 0 , coincide with the former?

\subsection{Properties of the finite viability kernel}

A first answer is given by applying Proposition 2.2 since $\lim _{h \rightarrow 0} \alpha(h)=0$, we have

$$
\bigcap_{h>0} \operatorname{Viab}_{G^{\alpha(h)}}(K)=\operatorname{Viab}_{G}(K)
$$


The following result gives a necessary and sufficient condition for $V_{i a b_{G_{h}^{\alpha(h)}}}\left(K_{h}\right)$ to be the reduction of $\operatorname{Viab}_{G^{\alpha(h)}}(K)$ to $X_{h}$ :

Let $G^{\alpha(h)}: X \rightsquigarrow X, G_{h}^{\alpha(h)}: X_{h} \rightsquigarrow X_{h}$ and $K_{h}$ a finite subset of $\operatorname{Dom}\left(G_{h}^{\alpha(h)}\right)$ defined as follows:

$$
\left\{\begin{array}{l}
G^{\alpha(h)}(x):=G(x)+\alpha(h) \mathcal{B} \\
K_{h}:=K \cap X_{h} \\
\forall x_{h} \in \operatorname{Dom}(G) \cap X_{h}: G_{h}^{\alpha(h)}\left(x_{h}\right):=G^{\alpha(h)}\left(x_{h}\right) \cap X_{h}
\end{array}\right.
$$

From definition of $\alpha(h), \forall x_{h} \in K_{h}, G_{h}^{\alpha(h)}\left(x_{h}\right) \neq \emptyset$.

Proposition 4.1 Let $G: X \rightsquigarrow X$ an upper semicontinuous set-valued map with closed values and $K$ a closed subset of $\operatorname{Dom}(G)$.

Let $r$ such that $\forall x \in \operatorname{Dom}\left(G^{r}\right) \cap X_{h}, G^{r}(x) \cap X_{h} \neq \emptyset$ :

$$
\operatorname{Dom}\left(G_{h}^{r}\right)=\operatorname{Dom}\left(G^{r}\right) \cap X_{h}
$$

Then

$$
\operatorname{Viab}_{G_{h}^{r}}\left(K_{h}\right) \subset \operatorname{Viab}_{G^{r}}(K) \cap X_{h}
$$

It coincides if and only if $\operatorname{Viab}_{G^{r}}(K) \cap X_{h}$ is a discrete viability domain of $K$ under $G^{r}$ :

$$
\forall x_{h} \in \operatorname{Viab}_{G^{r}}(K) \cap X_{h}, G^{r}\left(x_{h}\right) \cap\left(\operatorname{Viab}_{G^{r}}(K) \cap X_{h}\right) \neq \emptyset
$$

Proof - From (37) we have to check that the two following statements

$$
\forall x_{h} \in \operatorname{Viab}_{G^{r}}(K) \cap X_{h}, \quad G^{r}\left(x_{h}\right) \cap\left(\operatorname{Viab}_{G^{r}}(K) \cap X_{h}\right) \neq \emptyset
$$

and

$$
\operatorname{Viab}_{G_{h}^{r}}\left(K_{h}\right)=\operatorname{Viab}_{G^{r}}(K) \cap X_{h}
$$

are equivalent.

Assume that 42 holds. Let $x_{h} \in \operatorname{Viab}_{G_{h}^{r}}\left(K_{h}\right)$. There exists $\vec{x}_{h} \in \vec{S}_{G_{h}^{r}}\left(x_{h}\right) \cap$ $\mathcal{K}_{h}$ viable in $K_{h} \subset K$. Since $G_{h}^{r}\left(x_{h}\right) \subset G^{r}\left(x_{h}\right), \vec{x}_{h} \in \vec{S}_{G^{r}}\left(x_{h}\right) \cap \mathcal{K}$ is viable in $K$. Then $x_{h} \in \operatorname{Viab}_{G}^{r}(K)$ and we obtain inclusion:

$$
\operatorname{Viab}_{G_{h}^{r}}\left(K_{h}\right) \subset \operatorname{Viab}_{G^{r}}(K) \cap X_{h} .
$$

On the other hand, $\operatorname{Viab}_{G^{r}}(K) \cap X_{h}$ is a discrete viability domain of $G^{r}$. Then from definition 2.1 and definition of $G_{h}^{r}$ it is also a finite viability domain of $G_{h}^{r}$ contained in $K_{h}$ and thus is contained in the finite viability kernel of $K_{h}$ under $G_{h}^{r}: \operatorname{Viab}_{G^{r}}(K) \cap X_{h} \subset \operatorname{Viab}_{G_{h}^{r}}\left(K_{h}\right)$. We obtain the opposite inclusion and prove that $(43)$ is true.

Conversely, if (43) holds, $\operatorname{Viab}_{G^{r}}(K) \cap X_{h}$ is the finite viability kernel of $K_{h}$ under $G_{h}^{r}$, it is obviously a finite viability domain of $G^{r}$.

\section{Remarks}


1 - Inclusion 40 is always true.

Let call $A:=\left\{x_{h} \in \operatorname{Viab}_{G^{r}}(K) \cap X_{h}, x_{h} \notin \operatorname{Viab}_{G_{h}^{r}}\right\}$

and $B:=\left\{x_{h} \in \operatorname{Viab}_{G^{r}}(K) \cap X_{h}, \mid G^{r}\left(x_{h}\right) \cap\left(\operatorname{Viab}_{G^{r}}(K) \cap X_{h}\right)=\right.$ $\emptyset\}$.

2 - It is easy to prove that $B \subset A$ and Proposition 4.1 says that if $B$ is empty, $A$ is empty too.

3 - If $A \neq \emptyset$, then all solutions $\vec{x}_{h}$ to the finite dynamical system starting from any point $x_{h}^{0} \in A$, must leaves $K_{h}$ after a finite number of steps, although $x_{0}$ belongs to the dicrete viability kernel of $K$ under $G^{r}$.

4 - If $B \neq \emptyset$, then all solutions $\vec{x}_{h}$ to the finite dynamical system starting from any point $x_{h}^{0} \in B$, leaves $K_{h}$ at the first step.

5 - If $x_{h}^{0} \in A$ and $x_{h}^{n}$ is the last element of a solution to the finite dynamical system, starting at $x_{h}^{0}$, which is still in $K_{h}$, then $x_{h}^{n} \in B$.

6 - If $G^{r}(x) \subset K, \forall x \in K$ then for all $x_{h} \in K_{h}, G^{r}\left(x_{h}\right) \cap K_{h} \neq \emptyset$.

Then inclusion 40 becomes an equality :

$$
K_{h}=\operatorname{Viab}_{G_{h}^{r}}\left(K_{h}\right)=\operatorname{Viab}_{G^{r}}(K) \cap X_{h} .
$$

\subsection{Approximation of the viability kernel of $K$ under $F$ by finite viability kernel in the Lipschitz Case}

When $G$ is a $k$-Lipschitz set-valued map, we cannot prove that in 40 , the inclusion becomes an equality. Nevertheless we have in the Lipschitz case an immediate and interesting information about points $x_{h} \in K_{h}$ which do not satisfy 41):

Proposition 4.2 Let $G: X \rightarrow X$ a $k$-Lipschitz set-valued map. Let $r \geq$ $\max (k, 1) \alpha(h)$. For all $x_{h} \in \operatorname{Viab}_{G^{r}}(K) \cap X_{h}$ such that

$$
G^{r}\left(x_{h}\right) \cap\left(\operatorname{Viab}_{G^{r}}(K) \cap X_{h}\right)=\emptyset
$$

then

$$
x_{h} \notin \operatorname{Viab}_{G}(K) .
$$

Proof - Let $x_{h} \in \operatorname{Viab}_{G}(K) \cap X_{h}$. From definition of the viability kernel, we have

$$
G\left(x_{h}\right) \cap \operatorname{Viab}_{G}(K) \neq \emptyset
$$

From definition of $\alpha(h)$,

$$
\left.\left(G\left(x_{h}\right) \cap \operatorname{Viab}_{G}(K)+\alpha(h) \mathcal{B}\right) \cap X_{h}\right) \neq \emptyset
$$


this implies that

$$
\left(G\left(x_{h}\right)+\alpha(h) \mathcal{B}\right) \cap\left(\operatorname{Viab}_{G}(K)+\alpha(h) \mathcal{B}\right) \cap X_{h} \neq \emptyset
$$

and for any $r \geq \max (k, 1) \alpha(h)$,

$$
\left(G\left(x_{h}\right)+r \mathcal{B}\right) \cap\left(\operatorname{Viab}_{G}(K)+\frac{r}{k} \mathcal{B}\right) \cap X_{h} \neq \emptyset
$$

Since $G$ is $k$-Lipschitz, we can apply Lemma 2.3 , and thenwe obtain:

$$
G^{r}\left(x_{h}\right) \cap\left(\operatorname{Viab}_{G^{r}}(K) \cap X_{h}\right) \neq \emptyset .
$$

In particular, if we apply this Proposition for $G=\Gamma_{\rho}$ we have:

$\forall x_{h} \in V i a b_{\Gamma_{\rho}^{\alpha(h)}}(K) \cap X_{h}$ such that $\Gamma_{\rho}^{\alpha(h)}\left(x_{h}\right) \cap\left(\operatorname{Viab}_{\Gamma_{\rho}^{\alpha(h)}}(K) \cap X_{h}\right)=\emptyset$, then

$$
x_{h} \notin \operatorname{Viab}_{\Gamma_{\rho}}(K)
$$

and since from (31) $\operatorname{Viab}_{F}(K) \subset \operatorname{Viab}_{\Gamma_{\rho}}(K)$

$$
x_{h} \notin \operatorname{Viab}_{F}(K)
$$

We can deduce the following approximation result when $K$ is a viability domain:

Corollary 4.1 Let $G: X \rightarrow X$ a k-Lipschitz set-valued map and $K$ a viability domain of $G$. Then

$$
\forall r \geq \max (k, 1) \alpha(h), \operatorname{Viab}_{G_{h}^{r}}\left(K_{h}\right)=K_{h} .
$$

Proof - Let $X_{h} \in K_{h} . x_{h}$ belongs to $\operatorname{Viab}_{G}(K) \cap X_{h}$ and from Proposition $4.2 G^{r}\left(x_{h}\right) \cap\left(\operatorname{Viab}_{G^{r}}(K) \cap X_{h}\right) \neq \emptyset$. Since $\operatorname{Viab}_{G}(K) \subset \operatorname{Viab}_{G^{r}}(K)=K$, we replace $\operatorname{Viab}_{G^{r}}(K)$ by $K$ and then we obtain:

$$
\left.G^{r}\left(x_{h}\right) \cap K_{h}\right) \neq \emptyset
$$

that is to say that $K_{h}$ is a viability domain of $G_{h}^{r}$.

However, we prove that when $h$ goes to 0 , if $\rho$ goes to 0 "slower" than $a(h)$, we can approximate the viability kernel of $K$ under $F$ by a sequence of finite viability kernels of reduction to $X_{h}$ of some larger extensions of $\mathbf{1}+\rho F$.

We look now for extension $G^{r}$ of $G$ such that any solution $\vec{\xi} \in \vec{S}_{G}\left(\xi^{0}\right)$ can be approached by solution $\overrightarrow{\xi_{h}} \in \vec{S}_{G_{h}^{r}}\left(\xi_{h}^{0}\right)$ 
Lemma 4.1 Let $G: X \rightsquigarrow X$ a k-Lipschitz set-valued map. Let $r \geq k \alpha(h)$.

Let $G^{r}: X \rightsquigarrow X$ the extension of $G$ :

$$
\forall x \in X, G^{r}(x):=G(x)+r \mathcal{B} .
$$

and consider $G_{h}^{r}: X_{h} \rightsquigarrow X_{h}$ the reduction of $G^{r}$ to $X_{h}$ :

$$
\left.G_{h}^{r}\left(x_{h}\right):=G^{r}\left(x_{h}\right)\right) \cap X_{h}, \quad \forall x_{h} \in X_{h} .
$$

If the following property holds true:

$$
\forall \xi \in G(x), \exists \xi_{h} \in G(x) \cap X_{h} \text { such that }\left\|\xi-\xi_{h}\right\| \leq \frac{r}{k}
$$

Then with any solution $\vec{\xi}:=\left(\xi^{n}\right)_{n} \in \vec{S}_{G}\left(\xi^{0}\right)$ to the discrete dynamical system:

$$
\xi^{n+1} \in G\left(\xi^{n}\right), \forall n \geq 0
$$

we can associate a solution $\vec{\xi}_{h}:=\left(\xi_{h}^{n}\right)_{n} \in \vec{S}_{G_{h}^{r}}\left(\xi_{h}^{0}\right)$ to the finite dynamical system:

$$
\xi_{h}^{n+1} \in G_{h}^{r}\left(\xi_{h}^{n}\right), \forall n \geq 0
$$

such that

$$
\left\|\xi_{h}^{n}-\xi^{n}\right\| \leq \frac{r}{k}, \forall n \geq 0
$$

Proof - Let $\xi^{0} \in X$ and $\vec{\xi} \in \vec{S}_{G}\left(\xi^{0}\right)$. From definition of $\alpha(h)$, since $r \geq k \alpha(h), \exists \xi_{h}^{0} \in\left(\left\{\xi^{0}\right\}+\frac{r}{k} \mathcal{B}\right) \cap X_{h}$. Assume that we found a sequence $\xi_{h}^{k}$ satisfying (47) and (48) until $k=n$.

Since $G$ is $k$-Lipschitz,

$$
G\left(\xi^{n}\right) \subset G\left(\xi_{h}^{n}\right)+k\left\|\xi^{n}-\xi_{h}^{n}\right\| \mathcal{B}
$$

and then, from 48,

$$
G\left(\xi^{n}\right) \subset G^{r}\left(\xi_{h}^{n}\right)
$$

Since $\xi^{n+1} \in G\left(\xi^{n}\right)$, from 45 , there exists $\xi_{h}^{n+1} \in G\left(\xi^{n}\right) \cap X_{h}$ such that

$$
\left\|\xi^{n+1}-\xi_{h}^{n+1}\right\| \leq \frac{r}{k}
$$

On the other hand we have

$$
\xi_{h}^{n+1} \in G\left(\xi_{h}^{n}\right)+k\left\|\xi^{n}-\xi_{h}^{n}\right\| \mathcal{B} \subset G_{h}^{r}\left(\xi_{h}^{n}\right) .
$$

Since $G\left(\xi_{h}^{n}\right) \cap X_{h} \subset G_{h}^{r}\left(\xi_{h}^{n}\right), \quad \forall n \geq 0$, then $\vec{\xi}_{h} \in \vec{S}_{G_{h}^{r}}\left(\xi_{h}^{0}\right)$.

This ends the proof of Lemma 4.1

We deduce the following result: 

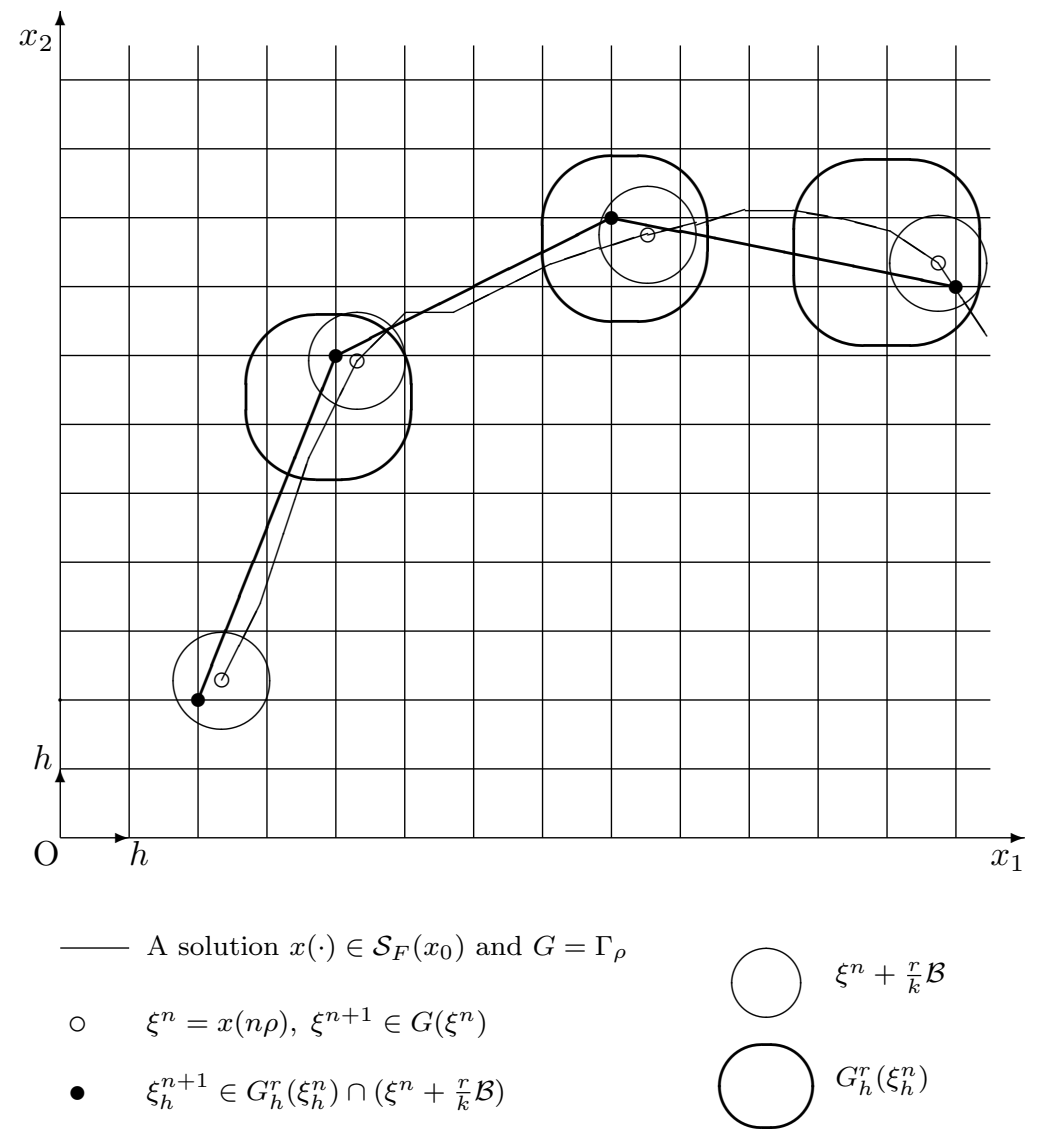

Figure 1: The Extension-Reduction Process

Corollary 4.2 Let $G: X \rightsquigarrow X$ a k-Lipschitz set-valued map satisfying property 45). Let $K$ a closed subset of $X$ Then, for all $r \geq k \alpha(h)$ we have:

$$
\operatorname{Viab}_{G}(K) \subset \operatorname{Viab}_{G_{h}^{r}}\left(K_{h}^{\frac{r}{k}}\right)+\frac{r}{k} \mathcal{B}
$$

Proof - From Lemma 4.1, for all $\xi^{0} \in \operatorname{Viab}_{G}(K)$, there exists $\vec{\xi} \in \vec{S}_{G}\left(\xi^{0}\right)$ viable in $K, \xi_{h}^{0} \in K_{h}^{\frac{r}{k}}$ and $\vec{\xi}_{h} \in \vec{S}_{G_{h}^{r}}\left(\xi_{h}^{0}\right)$ viable in $\left.\left(K_{h}^{\frac{r}{k}}\right)\right)$. By definition of the discrete viability kernel,

$$
\xi_{h}^{0} \in \operatorname{Viab}_{G_{h}^{r}}\left(K_{h}^{\frac{r}{k}}\right)
$$

and since $\left\|\xi^{0}-\xi_{h}^{0}\right\| \leq \frac{r}{k}$, we have

$$
\operatorname{Viab}_{G}(K) \subset \operatorname{Viab}_{G_{h}^{r}}\left(K_{h}^{\frac{r}{k}}\right)+\frac{r}{k} \mathcal{B}
$$

The reduction process satisfies the following property: 
Lemma 4.2 For any closed subset $D \subset X$, and any decreasing sequence of closed subsets $D_{\rho}$ such that $D=\bigcap_{\rho>0} D_{\rho}$, we have:

$$
D=\limsup _{\rho, h \rightarrow 0}\left(\left(D_{\rho}+\alpha(h) \mathcal{B}\right) \cap X_{h}\right)
$$

If $D$ is satisfies the property: $\forall x \in D, \exists x_{h} \in D \cap X_{h}:\left\|x-x_{h}\right\| \leq \alpha(h)$, then

$$
D=\limsup _{h \rightarrow 0}\left(D \cap X_{h}\right)
$$

Proof - Proof of second statement is immediate. We just prove the first equality.

Let $x \in \lim \sup _{\rho, h \rightarrow 0}\left(D_{\rho}+\alpha(h) \mathcal{B}\right) \cap X_{h}$. There exists $\rho_{n}, h_{n}$ converging to zero and $x_{\rho_{n} h_{n}} \in\left(D_{\rho_{n}}+\alpha\left(h_{n}\right) \mathcal{B}\right) \cap X_{h_{n}} \subset D_{\rho_{n}}+\alpha\left(h_{n}\right) \mathcal{B}$ which converges to $x$ when $n$ converge to $\infty$. Then $x \in \bigcap_{\rho \rightarrow 0} \bigcap_{h \rightarrow 0}\left(D_{\rho}+\alpha(h) \mathcal{B}\right)=D$.

Conversely, let $x \in D$. Since $D=\bigcap_{\rho \rightarrow 0} D_{\rho}$, there exists a sequence $x_{\rho}$ which converges to $x$. From definition of $\alpha(h)$, there exists $y_{\rho h} \in\left(D_{\rho}+\alpha(h) \mathcal{B}\right) \cap X_{h}$ such that $\left\|y_{\rho h}-x_{\rho}\right\| \leq \alpha(h)$ and $\lim _{\rho, h \rightarrow 0} y_{\rho h}=x$.

\subsection{Approximation of $\operatorname{Viab}_{F}(K)$ by Viability Kernel of fi- nite subsets $\operatorname{Viab}_{\Gamma_{h}^{r}}\left(K_{h}^{r}\right)$}

Now we can state the following result:

Theorem 4.1 Let $F: X \rightsquigarrow X$ a Marchaud and l-Lipschitz set-valued map, $K$ a closed subset of $\operatorname{Dom}(F)$ satisfying the boundedness condition:

$$
M:=\sup _{x \in K} \sup _{y \in F(x)}\|y\|<\infty
$$

Let $G_{\rho}:=\mathbf{1}+\rho F, \Gamma_{\rho}:=\mathbf{1}+\rho F+\frac{M l}{2} \rho^{2} \mathcal{B}$ and we note $k=1+\rho l$.

Let $h>0, X_{h}$ a reduction of $X$ and $\alpha(h)$ defined by (33).

Assume that $\rho$ and $h$ are choosen such that:

$$
\alpha(h) \leq \frac{M l}{2} \rho^{2}
$$

Let $\Gamma_{\rho}^{k M l \rho^{2}}: X \rightarrow X$ and $\Gamma_{\rho h}^{k M l \rho^{2}}: X_{h} \rightarrow X_{h}$ defined as follows :

$$
\begin{gathered}
\Gamma_{\rho}^{k M l \rho^{2}}(x):=\Gamma_{\rho}(x)+k M l \rho^{2} \mathcal{B} \\
\Gamma_{\rho h}^{k M l \rho^{2}}\left(x_{h}\right):=\Gamma_{\rho}^{k M l \rho^{2}}\left(x_{h}\right) \cap X_{h}
\end{gathered}
$$

Then:

$$
\operatorname{Viab}_{F}(K)=\limsup _{\rho, h \rightarrow 0}\left(\operatorname{Viab}_{\Gamma_{\rho}}(K)+\alpha(h) \mathcal{B}\right) \cap X_{h}
$$

and

$$
\operatorname{Viab}_{F}(K)=\limsup _{\rho, h \rightarrow 0} \operatorname{Viab}_{\Gamma_{\rho h}^{k M l \rho^{2}}}\left(K_{h}^{M l \rho^{2}}\right)
$$


Proof - From Theorem 3.2

$$
\operatorname{Viab}_{F}(K)=\limsup _{\rho \rightarrow 0} \operatorname{Viab}_{\Gamma_{\rho}}(K)
$$

The sequence of embeded subsets $\operatorname{Viab}_{\Gamma_{\rho}}(K)$ converges to $\operatorname{Viab}_{F}(K)$ when $\rho$ decreases to zero. Then applying Lemma 4.2 , we obtain the first equality (53):

$$
\operatorname{Viab}_{F}(K)=\limsup _{\rho, h \rightarrow 0}\left(\operatorname{Viab}_{\Gamma_{\rho}}(K)+\alpha(h) \mathcal{B}\right) \cap X_{h}
$$

To prove the second equality (54), we apply Corollary 4.2 with $G=\Gamma_{\rho}$.

We have to check first that assumption (52) implies the thickness condition 45) of Corollary 4.2 .

indeed

$$
\forall \xi \in \Gamma_{\rho}(x), \exists \xi^{\prime} \in x+\rho F(x) \text { such that }\left\|\xi^{\prime}-\xi\right\| \leq \frac{M l}{2} \rho^{2}
$$

From the definition of $\alpha(h)$

$$
\exists \xi_{h}^{\prime} \in X_{h} \text { such that }\left\|\xi_{h}^{\prime}-\xi^{\prime}\right\| \leq \alpha(h)
$$

Since from 52 ,

$$
(x+\rho F(x)+\alpha(h) \mathcal{B}) \cap X_{h} \subset\left(x+\rho F(x)+\frac{M l}{2} \rho^{2} \mathcal{B}\right) \cap X_{h}=\Gamma_{\rho}(x) \cap X_{h}
$$

Then we proved that $\forall \xi \in \Gamma_{\rho}(x), \exists \xi_{h}^{\prime} \in \Gamma_{\rho}(x) \cap X_{h}$ such that $\left\|\xi_{h}^{\prime}-\xi\right\| \leq$ $\left\|\xi_{h}^{\prime}-\xi^{\prime}\right\|+\left\|\xi^{\prime}-\xi\right\| \leq M l \rho^{2}$

We are able now to apply Corollary 4.2 with $r=k M l \rho^{2}$ and thus we obtain:

$$
V i a b_{\Gamma_{\rho}}(K) \subset V i a b_{\Gamma_{\rho h}^{k M l \rho^{2}}}\left(K_{h}^{M l \rho^{2}}\right)+M l \rho^{2} \mathcal{B}
$$

which implies that

$$
\operatorname{Viab}_{F}(K) \subset \limsup _{\rho, h \rightarrow 0}\left(\operatorname{Viab}_{\Gamma_{\rho h}^{k M l \rho^{2}}}\left(K_{h}^{M l \rho^{2}}\right)+M l \rho^{2} \mathcal{B}\right)
$$

and then

$$
\operatorname{Viab}_{F}(K) \subset \limsup _{\rho, h \rightarrow 0} V i a b_{\Gamma_{\rho h}^{k M l \rho^{2}}}\left(K_{h}^{M l \rho^{2}}\right)
$$

To prove the opposite inclusion, we observe that

$$
\Gamma_{\rho h}^{k M l \rho^{2}}=\mathbf{1}+\rho F+\left(\frac{M l}{2} \rho^{2}+k M l \rho^{2}\right) \mathcal{B}
$$

and then we have

$$
\operatorname{Graph}\left(\frac{\Gamma_{\rho h}^{k M l \rho^{2}}-\mathbf{1}}{\rho}\right) \subset \operatorname{Graph}(F)+\left(\frac{3}{2}+\rho l\right) M l \rho \mathcal{B}
$$


and if we assume for instance that $\rho l \leq \frac{1}{2}$,

$$
\left(\frac{3}{2}+\rho l\right) M l \rho \leq 2 M l \rho
$$

Then,

$$
\left.\forall \epsilon>0, \exists \rho_{\epsilon}>0 \text { such that } \forall \rho \in\right] 0, \rho_{\epsilon}[: 2 M l \rho \leq \epsilon
$$

and

$$
\left.\exists h_{\epsilon, \rho}>0 \text { such that } \forall h \in\right] 0, h_{\epsilon, \rho}\left[,: \alpha(h) \leq \frac{M l}{2} \rho^{2}\right.
$$

The Convergence Theorem 3.1 implies that:

$$
\limsup _{\rho, h \rightarrow 0} V i a b_{\Gamma_{\rho h}^{k M l \rho^{2}}}\left(K_{h}^{M l \rho^{2}}\right) \subset V i a b_{F}(K) .
$$

and with 55 we proved the equality

$$
\limsup _{\rho, h \rightarrow 0} V i a b_{\Gamma_{\rho h}^{k M l \rho^{2}}}\left(K_{h}^{M l \rho^{2}}\right)=\operatorname{Viab}_{F}(K)
$$

\subsection{Conclusion : a numerical method for computing via- bility kernel}

These results allow us to look for numerical approximation of the viability kernel of $K$ under $F$ associated with the initial differential inclusion (1):

$$
\dot{x}(t) \in F(x(t)), \text { for almost all } t \geq 0 .
$$

We consider the discrete explicit scheme:

$$
\left\{\begin{array}{l}
x^{n+1} \in x^{n}+\rho F\left(x^{n}\right)+2 M l \rho^{2} \mathcal{B}, \forall n \geq 0, \\
x^{0}=x_{0} \in K
\end{array}\right.
$$

We recall that the condition

$$
\left(x_{h}+\rho F\left(x_{h}\right)+r \mathcal{B} \cap X_{h} \neq \emptyset\right.
$$

will be true if $\rho$ and $h$ satisfy the condition:

$$
r=2 M l \rho^{2} \geq \alpha(h)
$$

witch is a stability condition meaning that the space discretization step has to be "smaller" than the time's one.

We set

$$
\Gamma_{\rho}(x):=x+\rho F(x)+\frac{M l}{2} \rho^{2} \mathcal{B}
$$




$$
G_{\rho}^{2 M l \rho^{2}}(x):=x+\rho F(x)+2 M l \rho^{2} \mathcal{B}
$$

From 13 in the discrete case and $(37)$ in the finite case we obtain:

$$
\begin{aligned}
K_{\rho}^{\frac{M l}{2} \rho^{2}, \infty} & :=\operatorname{Viab}_{\Gamma_{\rho}}(K) . \\
K_{\rho h}^{\frac{M l}{2} \rho^{2}, \infty} & :=\operatorname{Viab}_{\Gamma_{\rho h}}\left(K_{h}\right) .
\end{aligned}
$$

but $K_{\rho h}^{\frac{M l}{2} \rho^{2}, \infty}$ can be empty, and

$$
\begin{gathered}
K_{\rho}^{2 M l \rho^{2}, \infty}:=V i a b_{G_{\rho}^{2 M l \rho^{2}}}(K) . \\
K_{\rho h}^{2 M l \rho^{2}, \infty}:=V i a b_{G_{\rho h}^{2 M l \rho^{2}}}\left(K_{h}^{M l \rho^{2}}\right) .
\end{gathered}
$$

but now, if $h>0$ and $\rho>0$ satisfy the condition (57), the finite viability kernel $K_{\rho h}^{2 M l \rho^{2}, \infty}$ is non empty.

Gathering general results we proved in preceeding sections, we have the following convergence properties of approximations of viability kernel of $K$ under $F$ with finite viability kernels computable in a finite number of steps:

Theorem 4.2 If $F$ is a Marchaud setvalued map, $K$ a compact subset of $X$, $h>0$ and $\rho>0$ satisfying the condition (57).

Then

$$
\left\{\begin{array}{l}
K_{\rho, h}^{\frac{M l}{2} \rho^{2}, \infty} \subset K_{\rho}^{\frac{M l}{2} \rho^{2}, \infty} \cap X_{h} \\
\lim _{h \rightarrow 0} K_{\rho}^{\frac{M l}{2}} \rho^{2}, \infty=K_{\rho}^{\infty} \\
\limsup _{\rho \rightarrow 0} K_{\rho}^{\infty} \subset \operatorname{Viab}_{F}(K)
\end{array}\right.
$$

Moreover, if $F$ is l-Lipschitz, then

$$
\limsup _{\rho, h \rightarrow 0} K_{\rho, h}^{2 M l \rho^{2}, \infty}=\operatorname{Viab}_{F}(K)
$$

\section{References}

[1] AUBin J.-P. \& CELiNA A. (1984) Differential Inclusions Springer-Verlag, Berlin

[2] AUBIN J.-P.\& FRANKOWSKA H. (1990) SEt-valued analysis. Birkhaüser.

[3] AUBin J.-P. Viability Theory. To appear.

[4] Berge C. (1966) Espaces Topologiques, Fonctions MultiVOQUEs Dunod, Paris

[5] BYRNES C.I. \& ISIDORI A. (1990) RÉgulation Asymptotique DE Systèmes non LinÉAires C.R.A.S., Paris, 309, 527-530 
[6] FiliPPOV A.F. (1967) Classical solutions of Differential EQUATIONS WITH MULTIVALUED RIGHT HAND SIDE. SIAM, J. on Control, 5, 609-621

[7] FRANKOWSKA H. (To appear) Set-valued Analysis and ConTROL THEORY. Birkhaüser.

[8] FRANKOWSKA H. \& QUINCAMPOIX M. (1991) FAST ViabiLITY Kernel Algorithm.

[9] HadDAD G. (1981) Monotone viable Trajectories for Functional Differential Inclusions. J.Diff.Eq., 42, 1-24

[10] QUINCAMPOIX M. (1990) Frontière DE DOMAines D'INVARIANCE ET DE VIABILITÉ POUR DES INCLUSIONS DifFÉRENTIELles AVEC CONTRAINTES. Comptes Rendus Académie des Sciences, Paris

[11] SAinT-PiERRE P. (1990) Approximation of Slow Solutions to Differential Inclusions. Applied Mathematics and Optimisation. 22, 311-330.

[12] SAINT-PIERRE P. (1991) Viability of Boundary of the ViABILITY KERNEL. To appear 